\title{
Synthesis of (-)-Oseltamivir
}

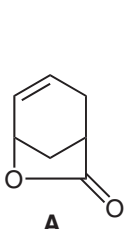

asymmetric allylic alkylation

1. $\left[\left(\eta^{3}-\mathrm{C}_{3} \mathrm{H}_{5} \mathrm{PdCl}\right)_{2}\right](2.5 \mathrm{~mol} \%)$

$\mathrm{PhthN}-\mathrm{SiMe}_{3}$ (1.5 equiv)

B $(7.5 \mathrm{~mol} \%)$, THF, $40^{\circ} \mathrm{C}$

2. $\mathrm{TsOH} \cdot \mathrm{H}_{2} \mathrm{O}, \mathrm{EtOH}, \Delta$

$84 \%(\mathrm{er}=99: 1)$
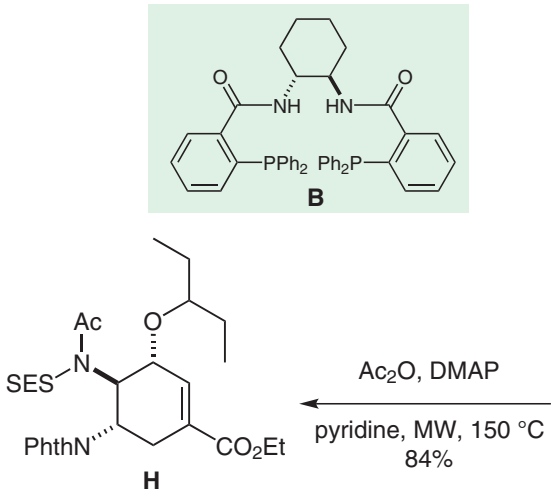

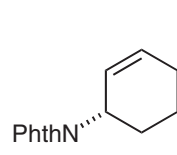

C
1. KHMDS ( 1.5 equiv)

2. $\mathrm{MCPBA}, \mathrm{NaHCO}_{3}$ (2 equiv)

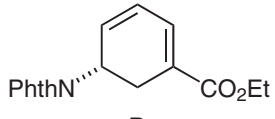

$\mathrm{PhSSO}_{2} \mathrm{Ph}, \mathrm{THF},-78^{\circ} \mathrm{C}$ $80 \%$

E (2 mol\%)

SES- $\mathrm{NH}_{2}$ (1.1 equiv) $\mathrm{Phl}\left(\mathrm{O}_{2} \mathrm{CCMe}_{3}\right)_{2}$ (1.3 equiv) $86 \%$

$\mathrm{MgO}$ (2.3 equiv) $\mathrm{PhCl}, 0^{\circ} \mathrm{C}$ to r.t.
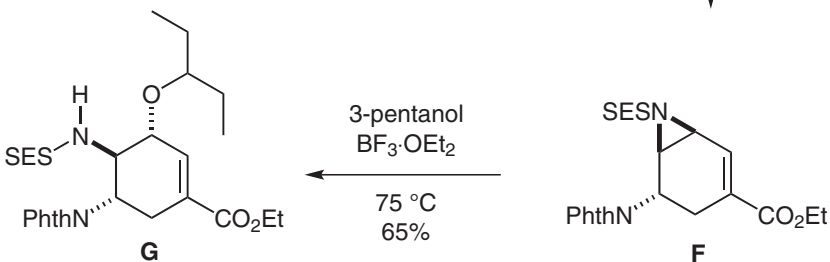

Products and

Potential Drugs

Key words

oseltamivir

tamiflu

aziridines

palladium

allylic substitution

nitrene

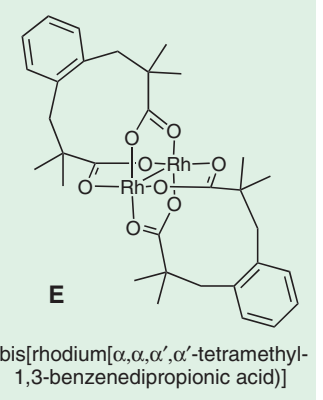

Significance: A very concise synthesis of (-)-oseltamivir (tamiflu) features a novel palladium-catalyzed asymmetric allylic alkylation reaction using a phthalimide nucleophile to directly open the racemic lactone $\mathbf{A}$.
Comment: The highly diastereo- and chemoselective nitrene insertion reaction leading to aziridine $\mathbf{F}$ was best accomplished using phenyliodonium bis(pivalate) as the oxidant, SES- $\mathrm{NH}_{2}$ as the nitrene source, and $\mathbf{E}$ as the catalyst. Copper catalysts showed poor chemoselectivity and silver or gold catalysts gave low reactivity. 